EESTI NSV TEADUSTE AKADEEMIA TOIMETISED. 29. KOIDE FOOSIKA * MATEMAATIKA. 1980, NR. 4

ИЗВЕСТИЯ АКАДЕМИИ НАУК ЭСТОНСКОИ ССР. ТОМ 29 ФИЗИКА * МАТЕМАТИКА, 1980, № 4

\title{
ДВЕ ЗАДАЧИ РАСПРЕДЕЛЕНИЯ ОГРАНИЧЕННЫХ ТОПЛИВНО-ЭНЕРГЕТИЧЕСКИХ РЕСУРСОВ
}

\author{
K. JAANIMAGI. KAKS PIIRATUD KUTUSE- JA ENERGIARESSURSSIDE JAOTAMISE OLESANNET \\ K. JAANIMAGI. TWO PROBLEMS OF THE DISTRIBUTION OF LIMITED FUEL ENERGY \\ RESOURCES
}

(Представил Н. Эпик)

B [i1] было показано, что при оптимизации топливно-энергетических балансов (ТЭБ) объединения районов следует учитывать четыре целевых функционала, подлежащих минимизации на области допустимых решений $D$, задаваемой неравенствами

$$
\left\{\sum_{i=1}^{n} \eta_{i l} x_{i l} \geqslant Q_{l}, \sum_{i=1}^{n} a_{i l}^{k} x_{i l} \leqslant Y_{l}^{k}, \sum_{l=1}^{m} x_{i l} \leqslant x_{i}\right\} .
$$

Целевой функционал $f_{1}(\bar{x})$ представляет собой суммарные приведенные затраты в топливно-энергетический комплекс (ТЭК); $f_{2}^{k}(\bar{x})-$ суммарные выбросы $k$-го загрязняющего вещества в район ТЭК; $f_{3}(\bar{x})$ - суммарные людские ресурсы ТЭК, которые не входят составной частью в $f_{1}(\bar{x}) ; f_{4}(\bar{x})$ - техническая ненадежность ТЭК.

В данной работе мы предлагаем для решения задачи оптимизации ТЭБ объединения $v$ районов с учетом этих целей применять метод, состоящий из 3 этапов:

1. Решая для каждого $u$-го района четыре задачи линейного программирования

$$
\begin{gathered}
f_{j}^{u_{\prime}}(\bar{x}) \rightarrow \min , \\
\bar{x}=\left\{x_{i l}\right\} \in D_{u},
\end{gathered}
$$

где $j=1,2,3,4 ; i=1,2, \ldots, n ; l=1,2, \ldots, m ; u$-индекс района, строим энергоэкономические характеристики $\left(Z_{1}{ }^{u}\right)$ ТЭБ района $\left[{ }^{2}\right]$, характеристики загрязнения $\left(Z_{2}{ }^{u}\right)$, трудовых затрат $\left(Z_{3}{ }^{u}\right)$ и ненадежности $\left(Z_{4}{ }^{u}\right)$. Эти характеристики представляют собой полиномы второго порядка, выражающие зависимость соответствующих целевых функционалов от количества эффективных видов топлива. 2. Решая методами из $\left[{ }^{3}\right]$ четыре многоцелевые задачи квадратич-
ного программирования

$$
Z_{j}^{u}(\bar{x}) \rightarrow \min , \quad u=1,2, \ldots, v,
$$




$$
\sum_{u=1}^{v} x_{i u}=x_{i}, \quad\left|x_{i u}\right| \leqslant x_{i}
$$

строим обобщенные критерии вида $\Phi_{j}=\sum_{u=1}^{v} \lambda_{u} E Z_{j}^{u}$.

3. Решая многоцелевую задачу квадратичного программирования

$$
\Phi_{j} \rightarrow \min , \quad j=1,2,3,4,
$$

при $\bar{x}$, удовлетворяющем (4), получаем оптимальные количества эффективных видов топлива по районам.

В процессе решения задачи оптимизации ТЭБ объединения районов в условиях неопределенности для всевозможных значений параметров строится платежная матрица, строкам которой соответствуют оптимальные решения детерминированных задач, а столбцам - обобщенные функционалы, полученные в процессе решения задачи (5). Оптимальное решение выбирается или как оптимальная смешанная стратегия первого игрока в фиктивной матричной игре с полученной матрицей $\left[{ }^{3,4}\right]$, или на основе методов из $\left[{ }^{5}\right]$. Применимы также методы оптимизации в условиях вероятностного характера исходной информации [ $\left.{ }^{3}\right]$.

Для распределения ограничений потребляемой мощности и электроэнергии по отраслям и предприятиям промышленности в [ $\left.{ }^{3}\right]$ разработан многоцелевой метод. В условиях вероятностного характера исходной информации задача распределения ограничений по отраслям имеет вид:

$$
\begin{gathered}
E\left(\sum_{i=1}^{N} c_{1 i} x_{i}\right) \rightarrow \max , \\
E\left(\sum_{i=1}^{N} c_{2 i}\left(x_{i}^{\max }-x_{i}\right)\right) \rightarrow \min , \\
E\left(\sum_{i=1}^{N}\left(W_{i}^{\mathrm{ycr}} / W_{i}^{\max }\right) \cdot\left(x_{i} / x_{i}^{\max }\right)\right) \rightarrow \min , \\
E\left(\sum_{i=1}^{N} c_{3 i}^{-1}\left(x_{i}^{\max }-x_{i}\right)\right) \rightarrow \min , \\
E\left(\sum_{i=1}^{N} c_{5 i} \cdot x_{i}^{\max } / x_{i}\right) \rightarrow \min , \\
E\left(\sum_{i=1}^{N} c_{6 i} \cdot x_{i}^{\max } / x_{i}\right) \rightarrow \min , \\
\sum_{i=1}^{N} x_{i}=X, \\
P\left(x_{i} \leqslant Q_{i}\right) \geqslant \varepsilon_{i} .
\end{gathered}
$$

Законы распределения параметров $c_{1 i}, c_{2 i}, c_{3 i}, c_{5 i}, c_{6 i}, W_{i}^{\max }, x_{i}{ }^{\max }$, $Q_{i}$ получаются на основе применения $\chi^{2}$-критерия для выборок статистических данных. В качестве математических ожиданий случайных коэффициентов целевых функционалов модели выбираются их выбороч- 
ные оценки. Стохастическая задача (6)-(13) приводится к детерминированной. Для решения на ЭВМ задач линейного программирования с целевыми функционалами, представляющими собой обобщенные функционалы многоцелевой задачи (6)-(13), а также для получения оптимальных смешанных стратегий матричных игр, соответствующих этой задаче, применялся пакет LPS-360. Решение задачи распределения ограничений потребляемой мощности и электроэнергии в условиях неопределенности проводилось в соответствии с теорией матричных игр.

Разработанный в данной работе метод оптимизации ТЭБ объединения районов позволяет учитывать неопределенность исходной информации и многоцелевой характер развития энергетического хозяйства. В процессе применения этого метода получаются оптимальные решения как по отдельным критериям оптимизации ТЭБ, так и по совокупности их.

\section{Л ИТ Е Р А Т Р А}

1. Я а н и м яг и К.. Изв. АН ЭССР, Физ. Матем., 28, № 4, 362-364 (1979).

2. В а й к Л. Э., Принципы и методы взаимосвязанной оптимизации топливно-энергетического хозяйства районов и страны, Автореф. докт. дис., М., 1976.

3. Я а н и м яги К. Э., Методы оптимальюого распределения ограниченных топливноэнергетических ресурсов между потребителями, Автореф. канд. дис., Л., 1980.

4. Методы и модели согласования иерархических решений (под ред. А. А. Макарова), Новосибирск, «Наука», 1979.

5. Беляев Л. С., Решение сложных оптимизационных задач в условиях неопределенности, Новосибирск, «Наука», 1978.

Ннститут термофизики и электрофизики Академии наук Эстонской ССР

Поступила в редакцию 11/VI 1980 